ACCEPTED MANUSCRIPT

\title{
Efficient second harmonic generation by para-nitroaniline embedded in electro-spun polymeric nanofibres
}

To cite this article before publication: Hugo Gonçalves et al 2018 J. Phys. D: Appl. Phys. in press https://doi.org/10.1088/1361-6463/aaa9da

\section{Manuscript version: Accepted Manuscript}

Accepted Manuscript is "the version of the article accepted for publication including all changes made as a result of the peer review process, and which may also include the addition to the article by IOP Publishing of a header, an article ID, a cover sheet and/or an 'Accepted

Manuscript' watermark, but excluding any other editing, typesetting or other changes made by IOP Publishing and/or its licensors"

This Accepted Manuscript is @ 2018 IOP Publishing Ltd.

During the embargo period (the 12 month period from the publication of the Version of Record of this article), the Accepted Manuscript is fully protected by copyright and cannot be reused or reposted elsewhere.

As the Version of Record of this article is going to be / has been published on a subscription basis, this Accepted Manuscript is available for reuse under a CC BY-NC-ND 3.0 licence after the 12 month embargo period.

After the embargo period, everyone is permitted to use copy and redistribute this article for non-commercial purposes only, provided that they adhere to all the terms of the licence https://creativecommons.org/licences/by-nc-nd/3.0

Although reasonable endeavours have been taken to obtain all necessary permissions from third parties to include their copyrighted content within this article, their full citation and copyright line may not be present in this Accepted Manuscript version. Before using any content from this article, please refer to the Version of Record on IOPscience once published for full citation and copyright details, as permissions will likely be required. All third party content is fully copyright protected, unless specifically stated otherwise in the figure caption in the Version of Record.

View the article online for updates and enhancements. 


\title{
Efficient second harmonic generation by para-nitroaniline
}

\author{
embedded in electro-spun polymeric nanofibres
}

\author{
Hugo Gonçalves ${ }^{1}$, Inês Saavedra ${ }^{1}$, Rute AS Ferreira ${ }^{2}$, PE Lopes $^{3}$, Etelvina de \\ Matos Gomes $^{1}$, Michael Belsley ${ }^{1}$ \\ ${ }^{1}$ Centre of Physics, University of Minho, Campus de Gualtar, 4710-057 Braga, \\ Portugal \\ 2 Department of Physics, CICECO - Aveiro Institute of Materials, University of \\ Aveiro, 3810-193 Aveiro, Portugal \\ ${ }^{3}$ Institute for Polymers and Composites/i3N, University of Minho, Guimarães, \\ Portugal \\ E-mail: belsley@fisica.uminho.pt
}

\begin{abstract}
Intense well polarized second harmonic light was generated by Poly(methyl methacrylate) nanofibres with embedded para-Nitroaniline nanocrystals. Subwavelength diameter fibres were electro-spun using a 1:2 weight ratio of chromophore to polymer. Analysis of the generated second harmonic light indicates that the para-Nitroaniline molecules, which nominally crystalize in the centrosymmetric space group, were organized into noncentrosymmetric structures leading to a second order susceptibility dominated by a single tensor element. Under the best deposition conditions, the nanofibrers display an effective nonlinear optical susceptibility approximately two orders of magnitude greater than that of potassium dihydrogen phosphate. Generalizing this approach to a broad range of organic molecules with strong individual molecular second order nonlinear responses, but which nominally form centrosymmetric organic crystals, could open a new pathway for the fabrication of efficient sub-micron sized second harmonic light generators.
\end{abstract}

PACS: 42.65.-k Nonlinear optics; 42.65.An Optical susceptibility; hyperpolarizability, 42.65.Ky Frequency conversion; harmonic generation; 42.70.Jk Polymers and organics 


\section{Introduction}

New materials with tailored linear and nonlinear optical (NLO) properties are actively being sought to extend the frontiers that limit many photonic devices. Currently the majority of commercial/nonlinear photonic components are based on inorganic materials [1]. Despite the successful development of many new inorganic crystalline materials over the past several decades, the applications of these materials is often limited by the magnitude of the available optical nonlinearities as well as the associated fabrication costs. In particular, efficient generation of second harmonic light by sub-micron sized systems is not routinely available from inorganic materials. Recently, several different processing approaches to extend the range of available nonlinear optical materials have been explored, including self-assembly [2,3], poling [4], lithography [5,6], molecular beam epitaxy [7] and electrospinning deposition [8-12]. Concurrently, researchers have developed a variety of alternative building blocks such as metallic nanoparticles[13], semi-conductors quantum dots [14], 2D-Materials [15] and tailored organic molecules [16].

In particular, organic systems have been widely investigated for nonlinear optical applications. They possess several attractive features including their low cost, fast, large nonlinear responses over a broad frequency range and tailorability [4,16]. A paradigmatic example is the organic molecule of paraNitroaniline $(p N A)$ [17-20]. It has a delocalized $\pi$-electron system and unsaturated bridge linking a donor amino group $\left(\mathrm{NH}_{2}\right)$ and an acceptor nitro group $\left(\mathrm{NO}_{2}\right)$. The delocalization of the $\pi$-electrons leads to an asymmetric charge distribution resulting in a strong molecular hyperpolarizability ( $\beta$ ) [21]. Unfortunately, because it crystallizes in a centrosymmetric space group $\left(P 2_{1} / n\right)$ [17], the bulk second order nonlinear response of $p$ NA crystals vanishes. This commonly occurs in donor-pi bridge-acceptor organic crystals: the dominant dipole-dipole interactions result in a pairwise, side-by-side antiparallel molecular alignment within the unit cell [22]. Experimentally, several different approaches have sought to achieve useful SHG when employing $p \mathrm{NA}$ as the active molecular building block [10,18,20,23]. These range from modifying the surface of $p$ NA crystals [18] to the incorporation of the organic dye within carbon nanotube structures [24].

Recently we reported observing strong SHG from $p$ NA embedded in electro-spun poly(L-lactic acid) (PLLA) polymer nanofibres [10]. Here we report on similar results obtained using Poly(methyl methacrylate) (PMMA) as the polymeric host. PLLA, being a chiral polymer, induces a higher degree of orientation alignment to the pNA nanocrystals within the electrospun fibres. The intensity of the dominant $\mathrm{X}$-ray diffraction peak corresponding to the [202] plane relative to that of the [111] or [311] planes in PLLA fibres is more than four times greater than what we observe for the PMMA fibres. Nonetheless, as 


\section{Methods}

\subsection{Materials}

Para-nitroaniline ( $p$ NA) was purchased from Sigma Chemical Co and used as received. Poly(methyl methacrylate) (PMMA, Mw 120000) was purchased from Alfa Aesar. The raw materials were dissolved in dimethyl formamide $(\mathrm{DMF})$ and toluene at a 1:2 volume ratio. $\mathrm{DMF}\left(\mathrm{C}_{3} \mathrm{H}_{7} \mathrm{NO}, 99.8 \%\right)$ and toluene $\left(\mathrm{C}_{6} \mathrm{H}_{5} \mathrm{CH}_{3}, 99.8 \%\right)$ were purchased from Sigma-Aldrich as used as received. The relative weight ratio of $p$ NA to PMMA in the final precursor solution was 1:2. The prepared solutions were stirred for several hours under ambient conditions prior to deposition by electrospinning.

\subsection{Nanofibre Production}

The polymeric nanofibres were electro-spun. The precursor solution was loaded into a syringe with its needle connected to the anode of a high voltage power supply (Spellmann CZE2000). To produce the in- 


\subsection{Material Characterization}

The morphology, size and shape of $p$ NA-PMMA nanofibrers was verified by using a Nova Nano SEM 200 Scanning Electron Microscope (SEM) operated at an accelerating voltage of $10 \mathrm{kV}$. A homebuilt Fluorescent Lifetime Image Microscope (FLIM) [27] was used as a complementary analysis of the morphology and alignment of the $p$ NA-PMMA nanofibres. FLIM analysis gives information about the individual SHG response of each nanofibre.

Crystallinity and crystallographic orientation of the $p$ NA nanocrystals inside the nanostructured fibres was studied by X-ray diffraction. The X-ray diffraction pattern of nanofibres was measured from $\theta-2 \theta$ scans recorded between 10 and $60^{\circ}$ on a Philips PW-1710 X-ray diffractometer using $\mathrm{Cu}-\mathrm{K}_{\alpha}$ radiation of wavelength $1.5406 \AA$. The lattice planes parallel to the substrate surface were determined from the $q$ vector perpendicular to the nanofibres mat plane and thus perpendicular to the longitudinal nanofibre axes.

A high-resolution Raman spectrometer, Horiba LabRAM HR Evolution confocal microscope using laser excitation at $532 \mathrm{~nm}(2.33 \mathrm{eV})$, was employed to characterize the local molecular order and orientation of the $p$ NA inside of the PMMA host matrix. A 100x objective lens was used to focus the laser onto the sample. Polarized Raman spectra were obtained at room temperature between $600-1800 \mathrm{~cm}^{-1}$.

The bright field microscopic images were recorded using an Olympus BX51 microscope $(10 \times, 50 \times, 100 \times$ objectives), in the transmission mode, equipped with a digital CCD camera (Retiga 4000R, QImaging) used to capture the microphotographs (exposure time: $0.1 \mathrm{~s}$ ) of the samples under white light illumination via a DC regulated illuminator (DC-950, Fiber-Lite). The dark field images were acquired by replacing the standard microscope condenser, used for bright field transmission mode, by the CytoViVa enhanced darkfield illumination system. The hyperspectral microscope images were obtained with a digital camera (IPX-2M30, Imperx) coupled to a spectrograph (V10E 2/3", Specim, $30 \mu \mathrm{m}$ slit, nominal spectral range of 400-1000 nm, and nominal spectral resolution of $2.73 \mathrm{~nm}$ ). A UV-IR Cut Filter (IF 486, Schneider 
Kreuznach) with transmission range from 390 to $690 \mathrm{~nm}$ was placed in front of the cameras. Each pixel field-of-view on the hyperspectral images corresponds to an area of $128 \times 128 \mathrm{~nm}^{2}$ on the sample plane.

\subsection{Polarimetry measurements}

The SHG response of the $p$ NA-PMMA nanofibres was analysed by polarimetry measurements using the experimental set-up shown schematically in figure 1 . The incident fundamental light at frequency $\omega$ was provided by a femtosecond Ti:Sapphire mode-locked laser (Coherent Mira 900F) pumped by a frequency doubled CW Neodymium-laser (Coherent Verdi 5W), with a 100 femtosecond (fs) temporal pulse width and a central wavelength of $800 \mathrm{~nm}$. The measurement consists in illuminating $p$ NA-PMMA nanofibres with linearly polarized fundamental light and analysing the polarization of the transmitted SHG field. An achromatic half-wave plate $\left(\lambda_{2}\right)$ is placed before a $50 \mathrm{~mm}$ focal length plano-convex lens, in order to continuously vary the polarization direction of the incident light $\left(0-360^{\circ}\right)$. The generated SHG field is collected by a microscope objective 100× Mitutoyo Plan Infinity-Corrected Long working distance objective $(\mathrm{NA}=0.7)$. A set of filters was used to eliminate the transmitted fundamental beam intensity and select the SHG field with a central frequency of $2 \omega$. A polarizer was used to analyse the polarization of the generated SHG light. The polarizer was fixed to be either parallel (q-p configuration) or perpendicular (q-s configuration) to the nanofibre's longitudinal axis. The analysed SHG field was recorded by focusing the beam onto a multimode fibre optic cable coupled to the entrance slit of a $0.3 \mathrm{~m}$ imaging spectrograph Andor Shamrock SR-303i, with $0.20 \mathrm{~nm}$ resolution.

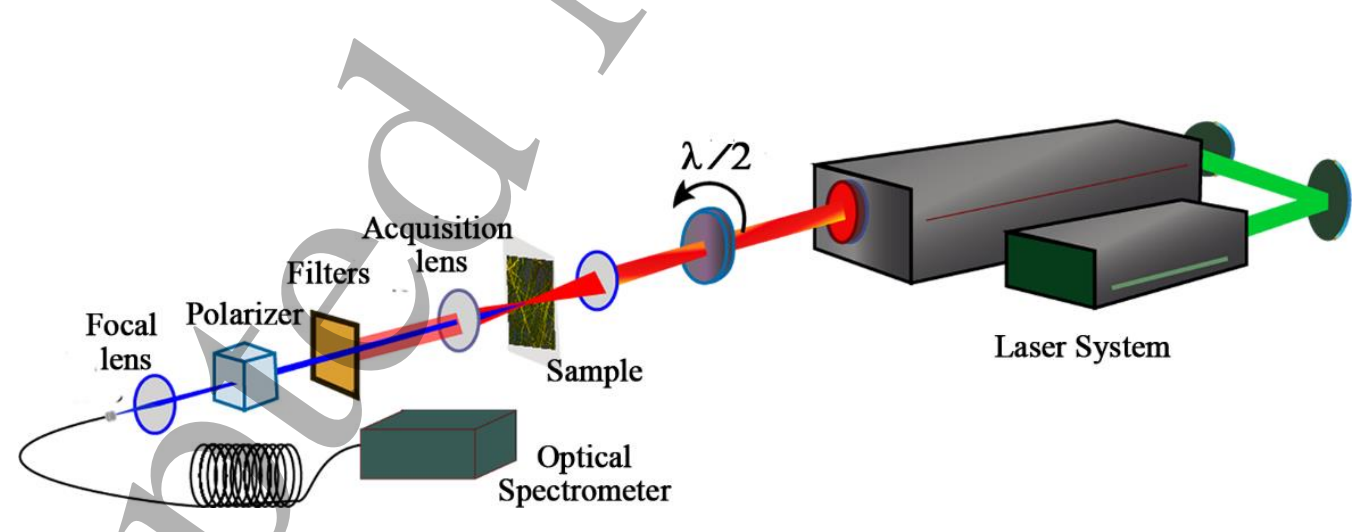

Figure 1. Polarimetry Setup. The sample is excited by a linearly polarized Ti:Sapphire laser beam. The polarization of the incident beam is continuously varied using an achromatic half-wave wave plate (WP $\lambda / 2$ ). After passing through a fixed polarization analyser the second harmonic signal is detected using a cooled CCD array coupled to a $0.3 \mathrm{~m}$ spectrograph.

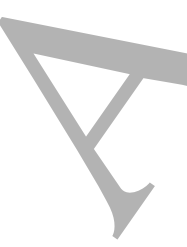




\section{Results and Discussion}

\subsection{Structural, Morphology and filled content analysis}

The morphology, size and shape of the $p$ NA-PMMA nanofibers were investigated using SEM as shown in figure 2 and summarized in table 1. One clearly sees that the electrospun fibres present a homogeneous cylindrical morphology with sub-micron diameters. A crucial aspect is the absence of $p$ NA crystals grown outside of the PMMA fibres confirming that the SHG signal comes from $p$ NA molecules located inside the polymeric matrix. No external beads are observed [28].

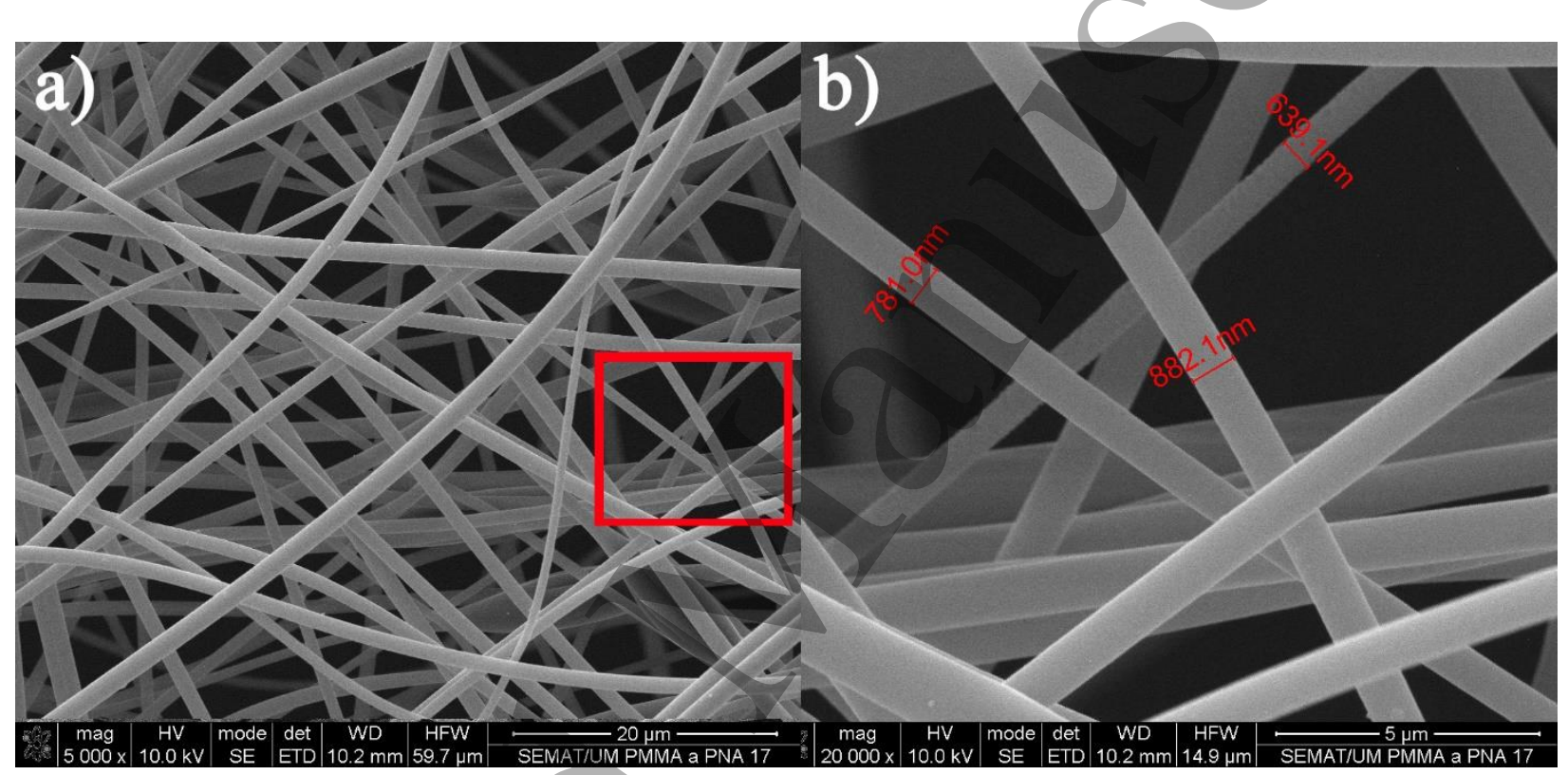

Figure 2. SEM image of $p$ NA-PMMA nanofibres (a) deposited by electrospinning using a deposition rate of $0.05 \mathrm{~mL} / \mathrm{h}$. (b) Detail of the selected area in the left image with an indication of a few selected nanofibre diameters.

The high spatial and time resolution of the FLIM microscopy setup allows the measurement of the SHG signal of each individual nanofibre (figure 3). Illuminating the nanofibres by the femtosecond laser with a central wavelength of $800 \mathrm{~nm}$ the resulting second harmonic response (SHG) near $400 \mathrm{~nm}$ is collected and recorded. Each pixel of the image in figure 3 is coloured and corresponds to a single decay profile at the respective pixel's spatial coordinate. The overall temporal response is represented in the histogram. The prompt SHG emission is characteristic of a scattering process in a well-defined temporal window with a width that is close to the instrumental response time of the detection system. This eliminates the possibility of other effects, such as fluorescence induced by the absorption of two photons. FLIM microscopy demonstrates that each individual $p$ NA-PMMA nanofibre presents a second order nonlinear response. 

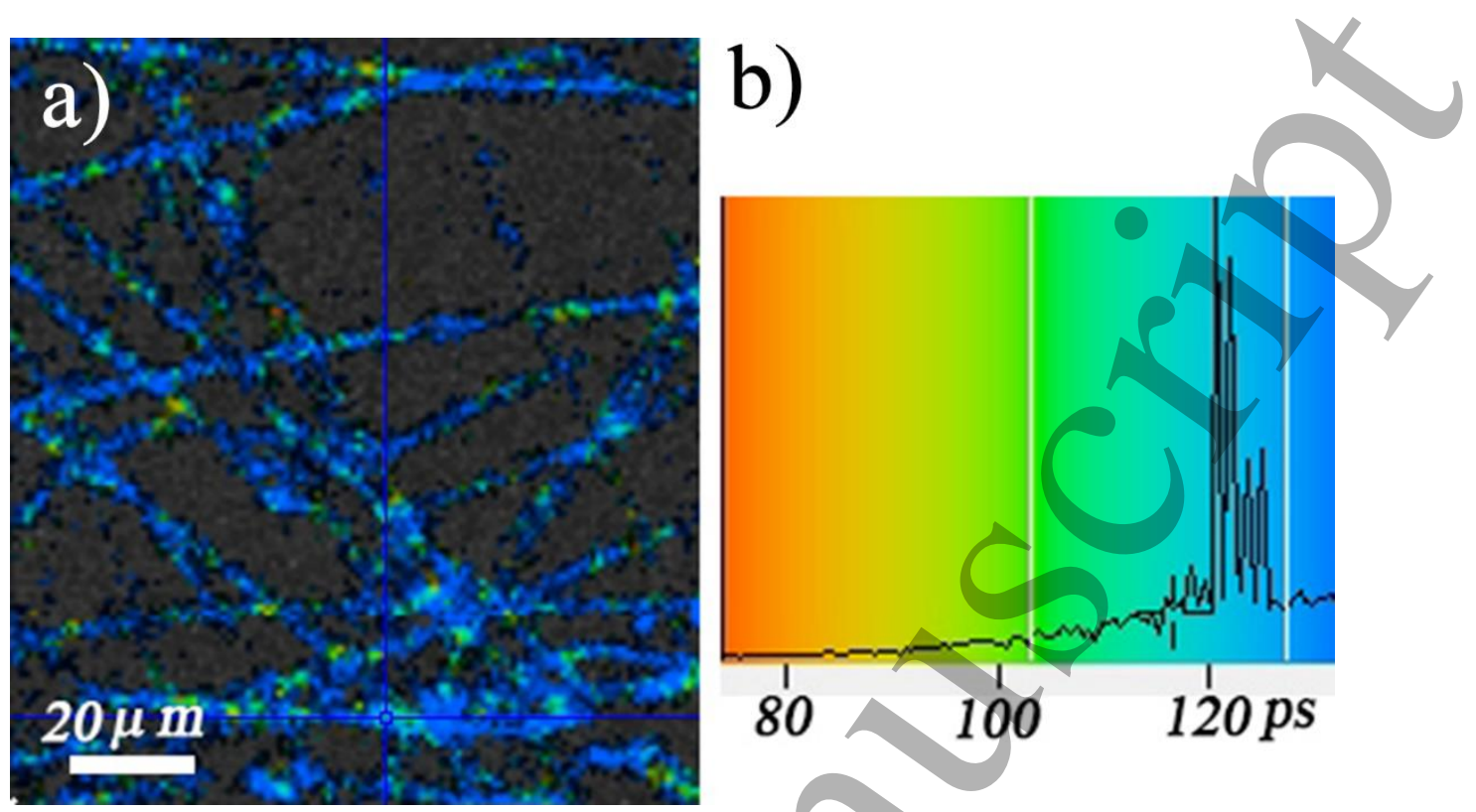

Figure 3. (a) Coloured FLIM microscope image of $p$ NA-PMMA nanofibres using a deposition rate of $0.05 \mathrm{~mL} / \mathrm{h}$. The image consists of $128 \times 128$ pixels (b) Colour encoded temporal histogram of the SHG response. The sharp peaks in the histogram, with widths close to the instrument response time of the system, are a characteristic of a prompt nonlinear effect.

The mean diameter of the $p$ NA-PMMA nanofibres tends to diminish as the deposition rate increases (table 1). Furthermore, the distribution of diameters broadens at higher deposition rates.

Table 1. Mean diameter of the $p$ NA-PMMA nanofibres as a function of the deposition rate.

\begin{tabular}{|cc}
$\begin{array}{c}\text { Deposition rate } \\
(\mathbf{m L} / \mathbf{h})\end{array}$ & $\begin{array}{c}\text { Mean Fibre Diameter } \\
(\mathbf{n m})\end{array}$ \\
\hline 0.05 & $786 \pm 45$ \\
0.40 & $594 \pm 64$ \\
0.75 & $578 \pm 140$ \\
\hline
\end{tabular}

$\mathrm{X}$-ray diffraction spectra acquired on $p$ NA-PMMA fibre mats for each of the deposition rates listed in table 1 are shown in figure 4 . The acquired diffraction patterns show evidence of well aligned nanocrystals dominated by a strong peak corresponding to the plane with the Miller indices (202) which is parallel to $p$ NA natural cleavage plane (101) [19]. After performing a baseline subtraction, a Lorentzian was fit to the (202) peak for the different deposition rates as shown in the inset of figure 4. 


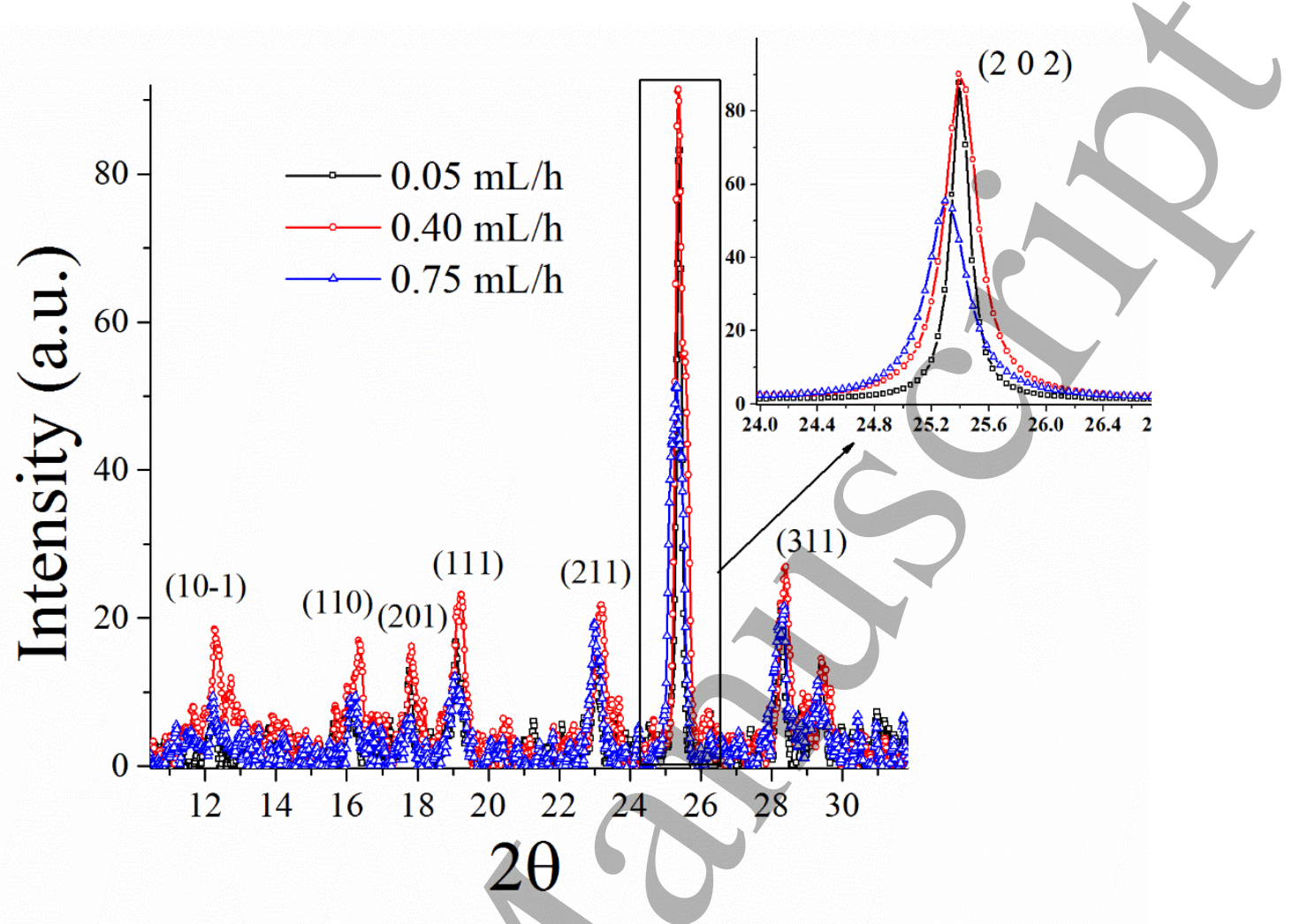

Figure 4. The X-Ray diffraction peaks of the PMMA nanofibres doped with $p$ NA, deposited at different rates. (Inset) Lorentzian fit to the peak corresponding to dominant peak with the Miller indices (202).

The Lorentzian fit allows us to quantify the broadening of the (202) diffraction peak as the deposition rate is increased. This broadening could be due either to a reduction in the mean size of the $p$ NA nanocrystals or an increase in strains to which they are subjected. When size effects dominate the broadening, the Scherrer equation [29] can be used to relate the width of the $h k l$ diffraction peak in radians, to the mean crystal size through the equation:

$$
\beta_{h k l}=\left(\frac{k \lambda}{D \cos (\theta)}\right)
$$

Here $\mathrm{D}$ is the crystal size in the direction perpendicular to the lattice planes, $\lambda$ is the wavelength of the radiation $(1.5406 \AA), \theta$ is the diffraction angle in radians and $k$ numerical factor frequently referred to as the crystallite-shape factor. In the absence of detailed shape information, $k=0.9$ is generally accepted as a good approximation. This allows us to estimate a minimum size for the $p$ NA crystals. 
Table 2. The experimental values of $\beta_{202}$ obtained by the Lorentzian peak fit of cleavage plane with the Miller indices (202) for the different deposition rate and the corresponding estimate for lower limit of the mean crystal size using equation 1 .

\begin{tabular}{ccc}
\hline $\begin{array}{c}\text { Deposition rate } \\
(\mathbf{m L} / \mathbf{h})\end{array}$ & $\begin{array}{c}\boldsymbol{\beta}_{202} \\
(\text { radians) }\end{array}$ & $\begin{array}{c}\text { Minimum } \boldsymbol{p} \mathbf{N A} \text { crystal size } \\
(\mathbf{n m})\end{array}$ \\
\hline 0.05 & 0.15 & 54.26 \\
0.40 & 0.28 & 29.07 \\
0.75 & 0.37 & 22.01 \\
\hline & & \\
\hline
\end{tabular}

The experimental values of $\beta_{202}$ and the corresponding minimum crystal sizes listed in table 2 together with the SEM results presented in table 1, suggest that nanofibres deposited at higher rates lead to thinner nanofibres containing smaller embedded $p$ NA nanocrystals. Generally, thinner nanofibres are the result of a fast and efficient solvent evaporation [9] as the nanofibres solidify on their way to the grounded collection substrate. Another possible consequence of the rapid solidification can be the increase of the surface tension generating micro-strains, which in turn broadens the diffraction peaks. For a deposition rate of $0.75 \mathrm{~mL} / \mathrm{h}$ the peak broadening is accompanied by a decrease of the peak area and maximum peak height. This suggests a lower degree of crystallinity accompanied by some combination of a decrease in the average size of the nanocrystals and an increase in the presence of micro-strains. The $0.05 \mathrm{~mL} / \mathrm{h}$ deposition rate leads to a significantly larger lower limit on the mean crystal size. At this deposition rate the average nanofibre diameter is larger, consistent with a slower evaporation rate which should lead to more uniform crystallization within the nanofibres. The preferential orientation corresponds to the plane with Miller indices (202) which is parallel to the natural cleavage plane (101). Theoretical calculations of surface quadratic susceptibilities in $p$ NA crystals [19] indicate that (101) and (202) surface terminations correspond to large quadratic susceptibilities and consequently strong surface nonlinear responses. Hyperspectral microscopy was used to ascertain whether the concentration of $p \mathrm{NA}$ varied significantly along the fibres. Figure 5a shows an optical image of $p$ NA fibres on a microscope slide. The fibre region was magnified (area marked with a rectangle) and the resulting hyperspectral image of a few $p$ NA fibres under white light excitation is depicted in figure 5c. The hyperspectral curves collected at a few selected points along the fibres (within pixels of size $128 \times 128 \mathrm{~nm}^{2}$ ) are spectrally uniform consistent with an overall homogenous concentration of embedded $p \mathrm{NA}$ molecules. We note that the intensity variations 
Figure 5. (a) Optical and (c) hyperspectral microscopy images of $p$ NA fibres in transmission dark-field mode (b) Hyperspectral spectrum measured within the single pixel areas (1-3 in c).

Raman spectroscopy was used to better understand the nature of the $p$ NA embedded within the polymeric fibres. Several peaks in the Raman spectrum have nearly the same frequencies in a thin sample of bulk crystalline $p$ NA and the nanofibres, both acquired at room temperature. The most prominent of these peaks are listed in table 3.

In figure 6, the Raman bands in bulk crystalline $p$ NA (solid line), and $p$ NA-PMMA nanofibres (dashed line) are shown for the spectral region between $600-1700 \mathrm{~cm}^{-1}$. Intense lines at $1282 \mathrm{~cm}^{-1}, 1314 \mathrm{~cm}^{-1}$ and 
$1338 \mathrm{~cm}^{-1}$ characterize the Raman spectrum of bulk $p$ NA crystals $[32,33]$. The most striking feature in figure 6 is the inexistence in the $p$ NA-PMMA nanofibre Raman spectra of the band centred at $1282 \mathrm{~cm}^{-1}$, which is the most intense peak in bulk crystalline $p$ NA. Harrand [32] has identified this peak as resulting from a superposition of the benezene ring symmetric stretching mode, $\omega_{3}$, with a combination line arising from another ring stretching mode and a torsion of the amino group, $\omega_{5}+\tau_{\mathrm{NH}_{2}}$. The hydrogen bonding between the nitro and amine groups of neighboring molecules induces $\pi$-electron charge concentration in the C-N bonds as well as between the bonds connecting unsubstituted carbons in the benzene ring. This produces a quinodal-like structure that leads to an enhanced Raman response. In contrast, the $1282 \mathrm{~cm}^{-1}$ peak is absent for $p \mathrm{NA}$ in solution where the lack of hydrogen bonding results in a more aromatic $\pi$-electron charge distribution. The absence of this prominent peak in the $p$ NA-nanofibres suggests that the normal hydrogen bonds between the nitro and amine groups are somehow weakened by the electro-spinning production technique. Alternatively, it might be evidence that the majority of $p$ NA molecules within the fibre are not incorporated into nanocrystals, but are rather embedded in a less organized structures. Nonetheless, the $\mathrm{x}$-ray diffraction spectra does indicate that a significant fraction of $p$ NA molecules do crystallize with their 202 planes oriented within the fibre mat.

Table 3. Raman frequency of the coincident peaks in bulk $p$ NA and the electro-spun nanofibres together with the identification of the corresponding vibrational mode ${ }^{1}$.

\begin{tabular}{cccl}
\hline $\begin{array}{c}\text { Raman Shift } \\
\left(\mathbf{c m}^{-1}\right)\end{array}$ & $\begin{array}{c}\text { Relative Intensity } \\
\text { (Nanofibres) }\end{array}$ & $\begin{array}{c}\text { Relative Intensity } \\
\text { (Thin Crystal) }\end{array}$ & Vibrational modes $^{\mathbf{a}}$ \\
\hline 860 & 0.34 & 0.24 & $\delta\left(\mathrm{NO}_{2}\right)$ \\
1109 & 0.34 & 0.12 & $v\left(\mathrm{C}-\mathrm{NO}_{2}\right)$ \\
1137 & 0.12 & 0.08 & $\varphi(13)$ \\
1178 & 0.12 & 0.07 & $\varphi(9 \mathrm{a})$ \\
1397 & 0.07 & 0.08 & $\varphi(19 \mathrm{~b})$ \\
1452 & 0.09 & 0.06 & $\varphi(19 \mathrm{a})$ \\
1507 & 0.11 & 0.06 & $v_{\text {as }}\left(\mathrm{NO}_{2}\right)$ \\
1598 & 0.20 & 0.08 & $\varphi(8 \mathrm{a})$ \\
\hline
\end{tabular}

${ }^{\mathrm{a}} v=$ stretching, $\delta=$ in-plane deformation, $\gamma=$ out-of-plane deformation, $\varphi=$ vibrations of benzene; nomenclature according to G. Varsanyi [30] .

The doublet $1313 / 1332 \mathrm{~cm}^{-1}$ band, that is characteristic of $p$ NA in solution and assigned predominately to symmetric $\mathrm{NO}_{2}$ stretch, is unexpectedly the strongest band in $p \mathrm{NA}$ nanofibre Raman spectra. For $p$ NA in solution, the relative intensities of the doublet varies with the solvent polarity. Interestingly, the observed ratio in the $p \mathrm{NA}$ nanofibres is similar to that of $p \mathrm{NA}$ in acetonitrile (a highly polar solvent), suggesting that $p$ NA molecules inside the PMMA matrix 
find themselves in a polar environment. This might indicate the presence of a residual electric field from the electro-spinning process that was frozen into the nanofibres as they solidified during the flight from the needle to the collecting drum. There is also a noticeable enhancement in intensity of the of $p$ NA nanofibre Raman peaks at $860 \mathrm{~cm}^{-1}$ which corresponds to $\mathrm{NO}_{2}$ in plane deformation ("scissors"); at $1109 \mathrm{~cm}^{-1}$ corresponding to a phenyl- $\mathrm{NO}_{2}$ stretch; and at $1598 \mathrm{~cm}^{-1}$ belonging to a phenyl ring stretching mode.

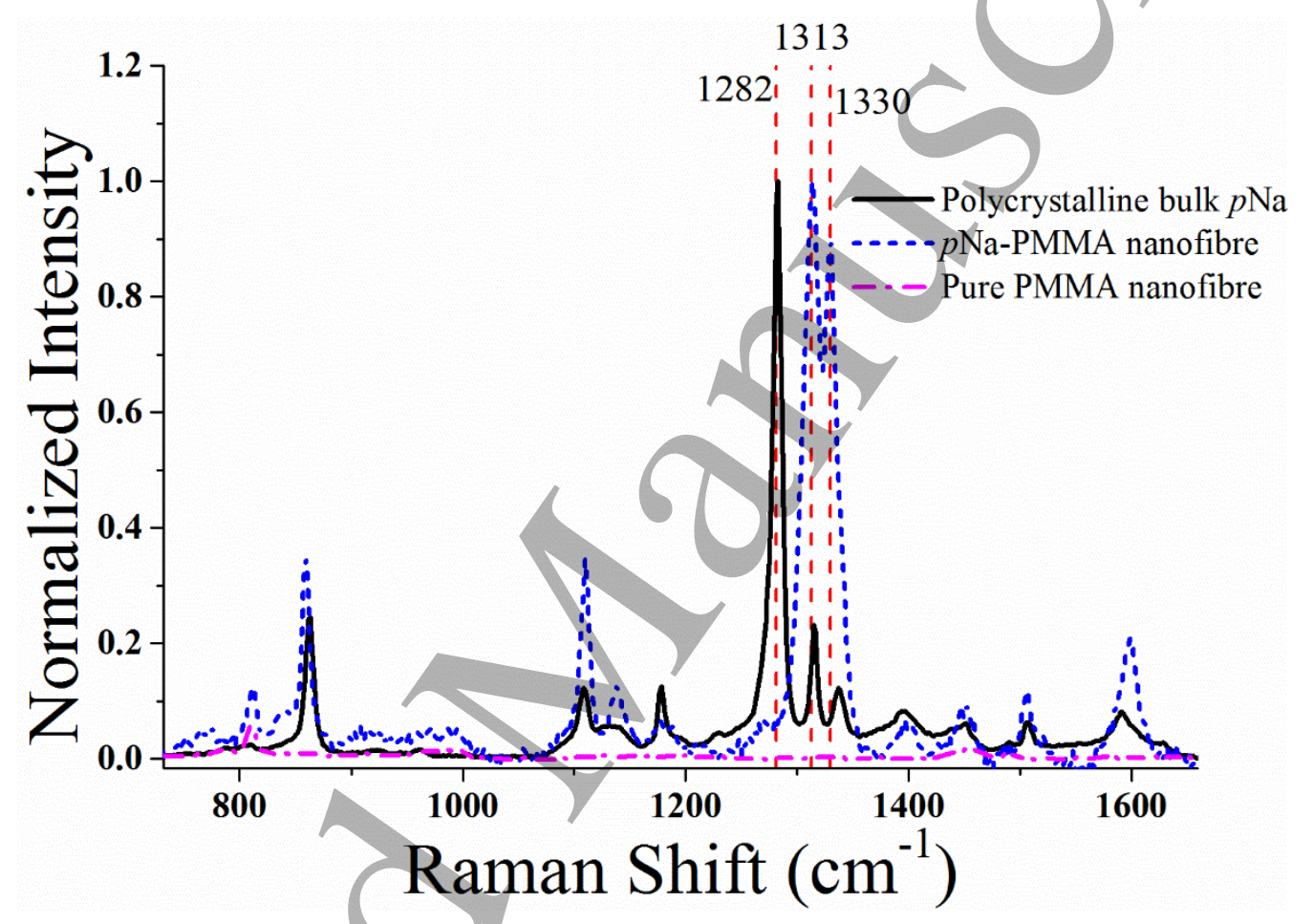

Figure 6. Raman spectra of a bulk sample of polycrystalline $p$ NA, a $p$ NA-PMMA nanofibre and a pure PMMA nanofibre (without $p N A$ ) between $600 \mathrm{~cm}^{-1}$ and $1700 \mathrm{~cm}^{-1}$. The two spectra containing $p$ NA were normalized to have the same maximum peak height, while the PMMA nanofibre spectrum is reported using the same scale as the $p$ NA-PMMA nanofibre spectrum.

The Raman spectra of un-doped PMMA nanofibres is strongly polarized as shown in figure 7. The polymer chains are found to preferentially align along the nanofibre direction. The peak at $810 \mathrm{~cm}^{-1}$ corresponds to the stretching mode of the $v(\mathrm{C}-\mathrm{O}-\mathrm{C})$ bond; its intensity decreases for crossed polarization, indicating an alignment of the PMMA scaffold and consequently an anisotropic environment for the embedded $p$ NA molecules. This may explain why the doublet intensity ratio observed for $p$ NA-PMMA nanofibres is similar to that of $p \mathrm{NA}$ in a polar solvent. 


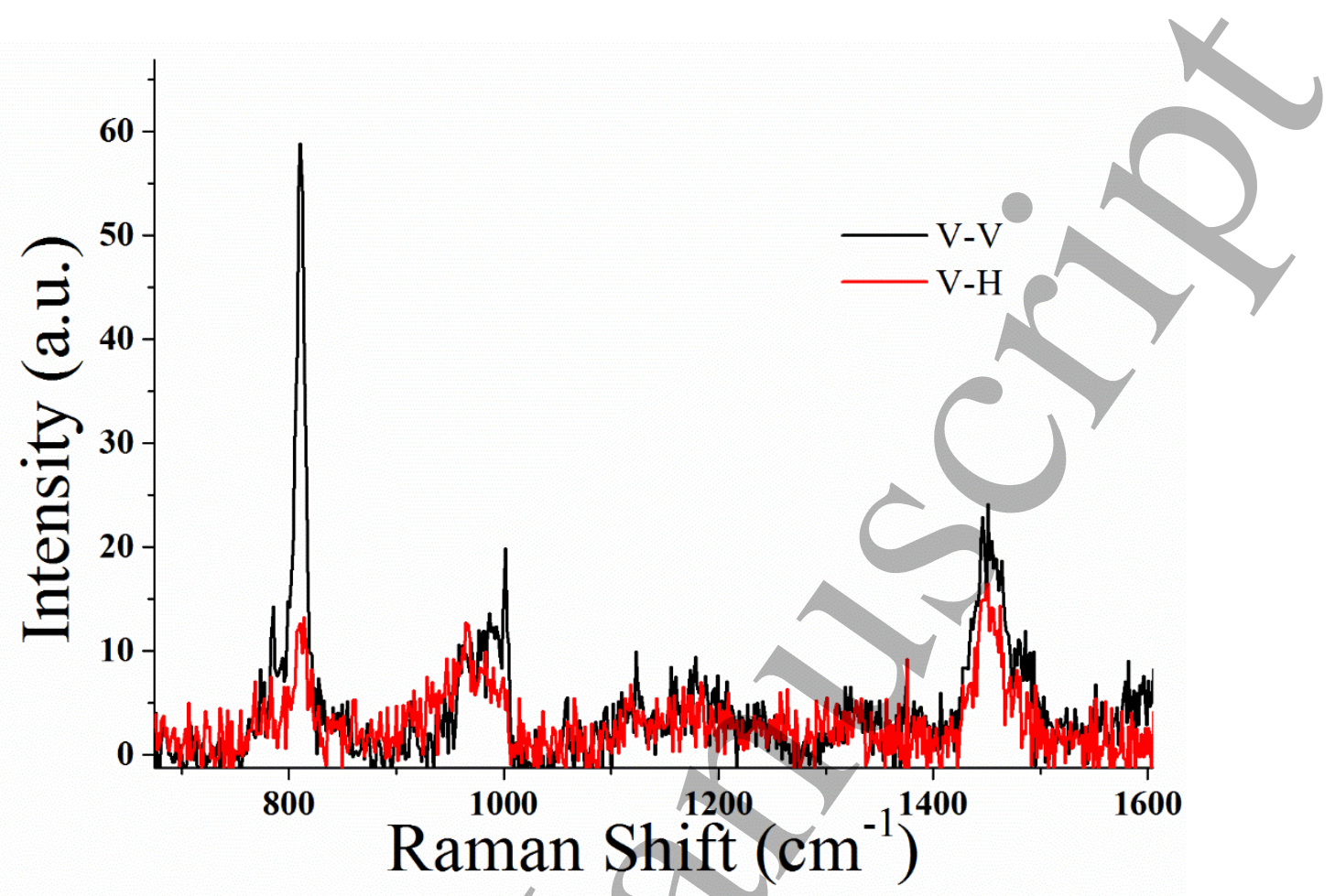

Figure 7. Raman spectra of PMMA nanofibres obtained with polarization configuration V-V and V-H.

\subsection{Second harmonic polarimetry}

The polarization properties of the second harmonic light was studied to assess the degree of orientational order of the $p$ NA molecules embedded within the nanofibres, using the experimental set-up of figure 1. While the polarization of the incident field varies continuously, the SHG field is collected in the so-called $q-p$ and $q-s$ configurations, i.e. with the analyser parallel and perpendicular to the longitudinal axis of the fibre respectively. A strong signal $\mathrm{SHG}$ resulted when the polarization of the incident fundamental light and the analyser were both aligned parallel to the selected fibre.

The SHG response of the $p$ NA-PMMA nanofibres shows a quadratic dependence as a function of the incident power (figure 8) as expected for a second order nonlinear effect. The r-squared coefficient for the quadratic fit is 0.998 . 


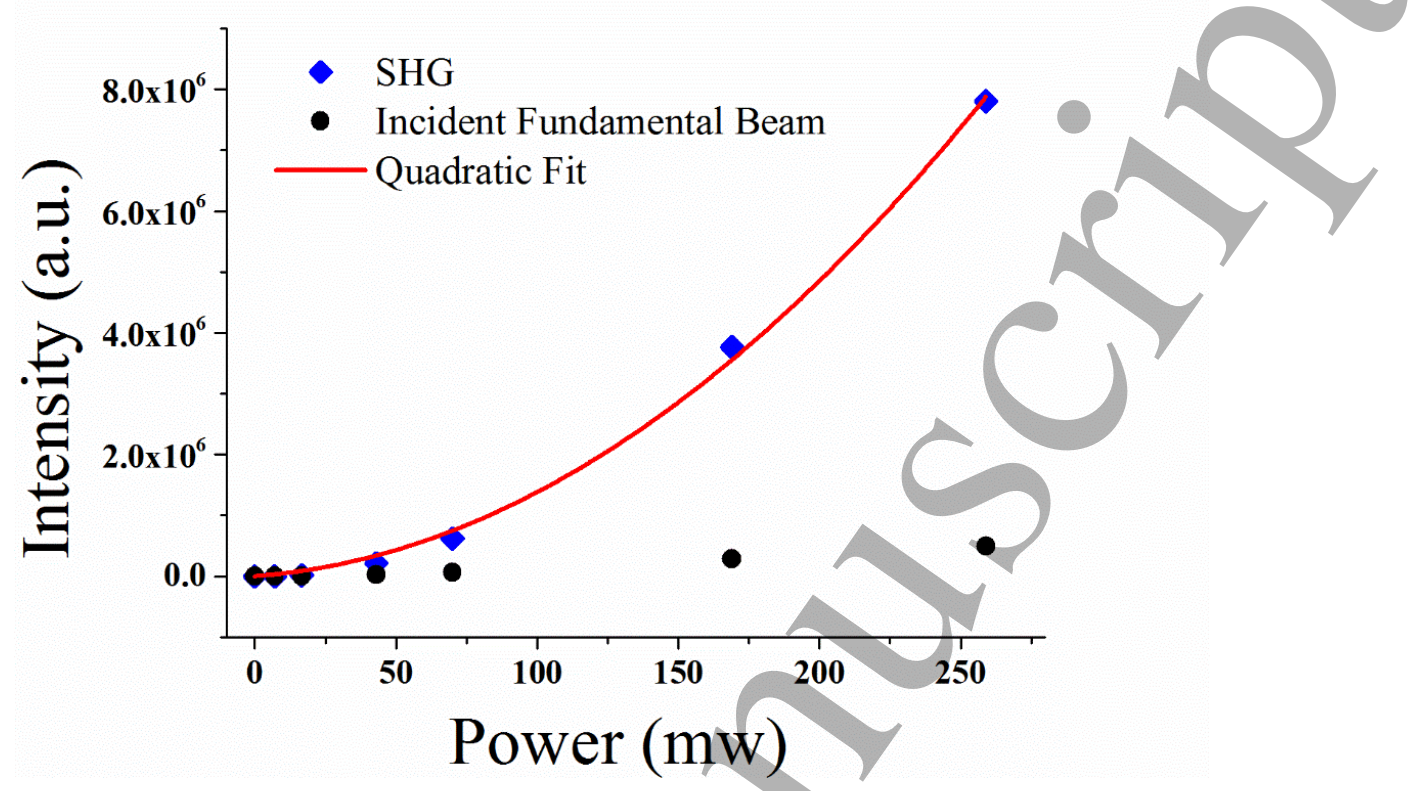

Figure 8. The SHG response of the $p$ NA-PMMA nanofibres using a deposition rate of $0.05 \mathrm{~mL} / \mathrm{h}$ as a function of the average incident power of the fundamental beam (repetition rate of $76 \mathrm{MHz}$ ).

The polarimetry curves display a polarization dependence that is characteristic of a SHG response dominated by a single diagonal second-order susceptibility tensor element, figure 9 . This is in contrast to the curves obtained for $p$ NA-PLLA fibres in which the q-s curves showed a lower but measureable cosine-squared like q-s response [10].

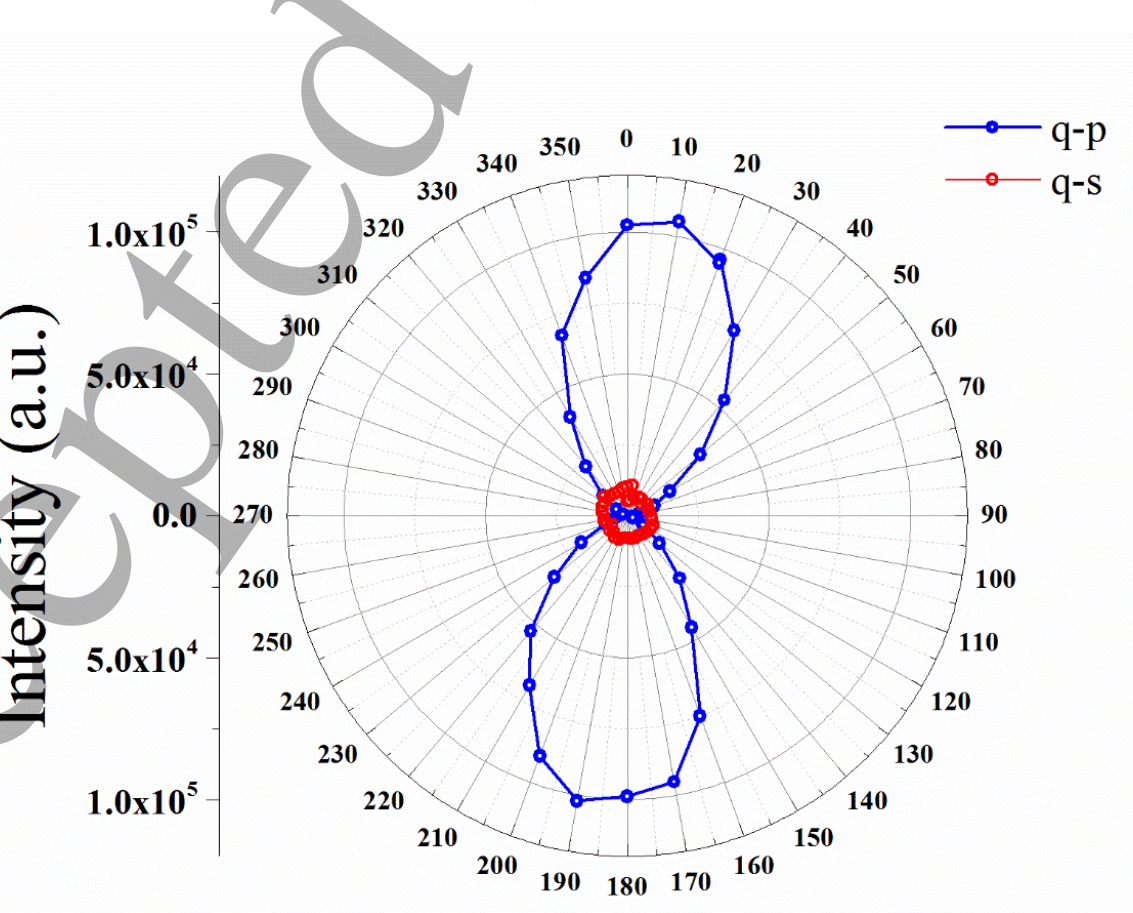


Figure 9. Polar plot of the SHG polarimetry data of $p$ NA-PMMA nanofibres produced using a deposition rate of $0.05 \mathrm{~mL} / \mathrm{h}$.

When the polarization direction of the incident field is continuously varied, the intensity of the SHG field decreases and reaches a minimum at $90^{\circ}$. This minimum can be a function of the orientation, micro-strain and/or size homogeneity of the nanocrystals inside the nanofibres [34]. The very good alignment of the nanocrystals leads to a nearly vanishing SHG field at cross polarization. The maximum intensity of the SHG radiation for the different deposition rates represented in Figure 10 was normalized to the same value in order to explore the variation with the deposition rate of the minimum intensity (at crossed polarization) of the SHG (inset). The lowest cross-polarization intensity corresponds to the $0.05 \mathrm{~mL} / \mathrm{h}$ deposition rate, consistent with the X-Ray diffraction data. This is the deposition rate that leads to a narrower diffraction peak indicating larger crystals or less micro-strain and consequently a larger degree of $p$ NA crystal alignment.

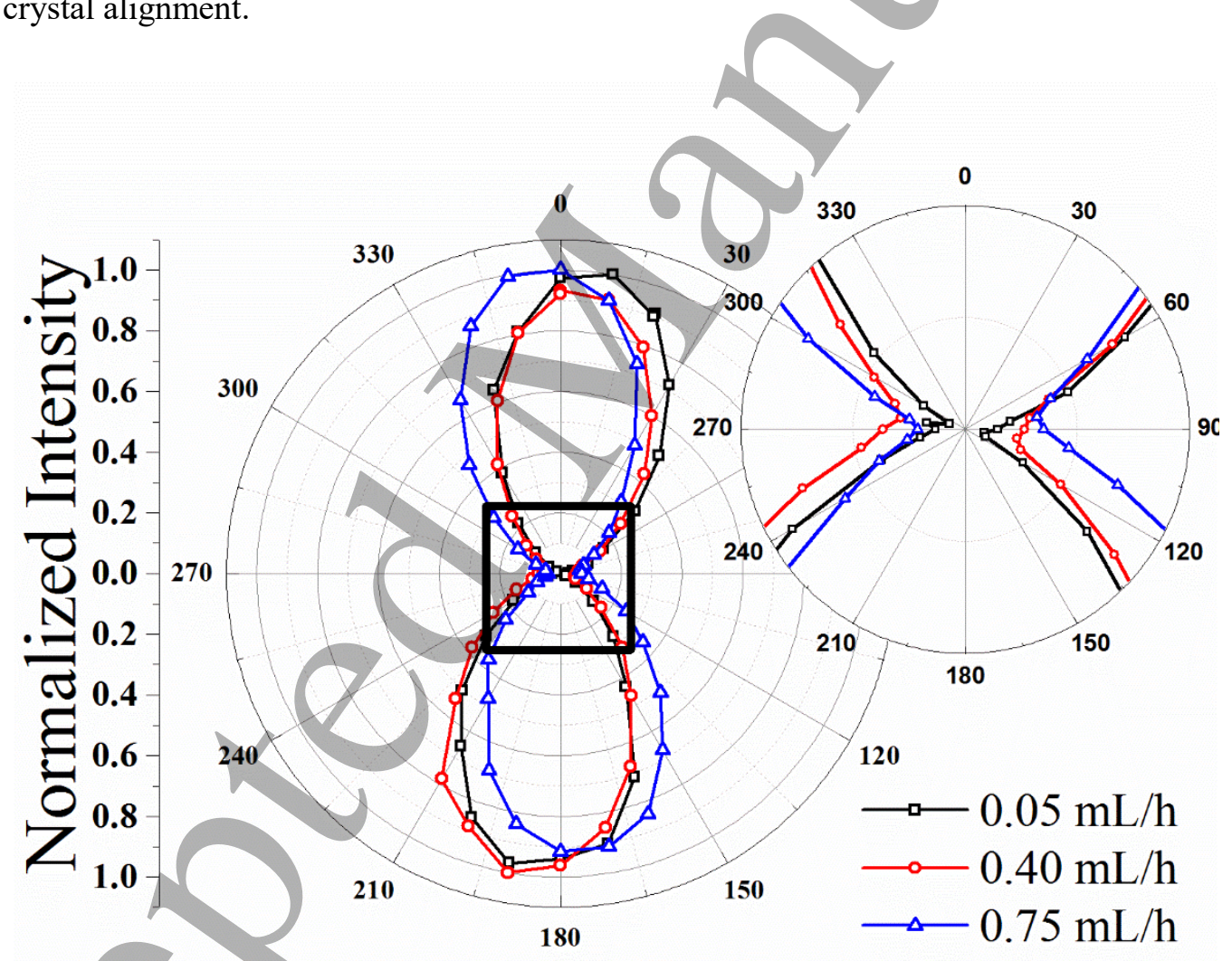

Figure 10. Polar plots of the normalized q-p SHG polarimetry data of PMMA nanofibres doped with $p \mathrm{NA}$ for the 3 different deposition rates. The close resemblance to a cosine squared polarization dependence is indicative of well-oriented SHG generators. (Inset) Variation of the minimum of SHG field near the position of crossed polarizer and analyser.

In figure 11 the second harmonic response of a $p$ NA embedded PMMA nanofiber mat taken at two different times separated by interval of one year is shown. Roughly $80 \%$ of the original 
Figure 11. Polar plots of the integrated SHG polarimetry data of PMMA nanofibres doped with $p$ NA produced at a deposition rate of $0.4 \mathrm{~mL} / \mathrm{h}$. The two polarimetry curves were taken at an interval of one year.

\subsection{Effective nonlinear optical coefficient}

In this section, we explore two potential explanations for observed second harmonic signal from the $p$ NA nanofibres. One possibility is that the signal is due to the surface polarization of the $p$ NA nanocrystals embedded within the nanofibres. We focus our attention on the nanofibres deposited at speed of 0.05 $\mathrm{mL} / \mathrm{h}$, which have the strongest observed SHG response. The SHG signal generated by a $4 \mathrm{~mm}$ thick potassium dihydrogen phosphate (KDP) crystal under the same excitation and detection conditions is used to calibrate the overall detection efficiency of our system.

The incident fundamental light consisted of pulses with and energy of approximately $0.2 \mathrm{~nJ}$, a FWHM duration of $100 \mathrm{fs}$ and a $1 / e^{2}$ diameter of $0.8 \mathrm{~mm}$. A $50 \mathrm{~mm}$ focal length plano-convex lens focused the fundamental beam onto the fibre or KDP crystal. We estimate that the diameter of the focused fundamental beam is roughly $65 \mu \mathrm{m}$ within the nanofibre or crystal with a corresponding confocal length, 
$b=k_{1} D^{2} / 4 \approx 1.2 \mathrm{~cm}$. Here $k_{1}$ is the magnitude of the wave vector for the fundamental beam in the medium, while D is the 1/e2 diameter at the focus. The expression for the SHG light intensity from a bulk crystal can be obtained from the solution of the nonlinear wave equation. When the confocal length of the incident fundamental beam is much greater than the crystal thickness the second harmonic power, $\mathcal{P}_{2 \omega}^{K D P}$, generated by the crystal is well described by solution obtained using the plane wave approximation [35,36]:

$$
\mathcal{P}_{2 \omega}^{K D P}=\frac{2 \omega^{2}}{\varepsilon_{0} n_{1}^{2} n_{2} c^{3}}\left[d_{e f f}\right]^{2} \frac{L^{2}}{\mathcal{A}} \operatorname{sinc}^{2}\left(\frac{\Delta k L}{2}\right) \mathcal{P}_{\omega}^{2}
$$

Here $\Delta k=2 k_{1}-k_{2}$ is the wave vector mismatch between the fundamental beam $\left(k_{1}\right)$ and the second harmonic beam $\left(k_{2}\right)$ within the medium, $\mathcal{A}$ is area of the focused beam waist and $d_{\text {eff }}$ is the effective nonlinear coefficient. For type I phase matching in KDP at the fundamental wavelength of $800 \mathrm{~nm}$, the appropriate second order coefficient is $d_{\text {eff }}=\sin \left(\theta_{p m}\right) d_{36}$, where the phase matching angle is $\theta_{p m}=44.9^{\circ}$ and $d_{36}=0.39 \mathrm{pm} \cdot \mathrm{V}^{-1}[36]$.

The birefringence of the KDP crystal induces a spatial walk-off between the wave-vector propagation and the direction of energy flow of the second harmonic beam that propagates as an extraordinary ray. In KDP at the phase matching orientation for $800 \mathrm{~nm}$ incident light, this walk-off angle is 29.5 mrads [37]. Furthermore, the dispersion of KDP imposes a group velocity mismatch of approximately $80 \mathrm{fs} / \mathrm{mm}$ [37] between the fundamental and second harmonic beams leading to a further temporal walk-off effect. Wang and Weiner [38] have developed a theoretical expression for the reduction in SHG efficiency in the presence of simultaneous spatial and temporal walk-off while taking into account the diffraction effects associated with Gaussian beam propagation. Using their expression we estimate that under our conditions these combined effects will reduce the SHG efficiency by roughly a factor of 3 from that predicted by the continuous plane-wave approximation implicit in equation (2) .

We couple the second harmonic light generated by the crystal or the nanofibre to the $0.3 \mathrm{~m}$ imaging spectrograph (Andor Shamrock SR-303i) via a multimode optical fibre bundle by employing a 100x infinity corrected long working length microscope objective from Mitutoyo (Plan Apo). This objective has a numerical aperture of 0.7 and an effective focal length of $2 \mathrm{~mm}$ both large enough to fully collect the second harmonic light generated by the crystal.

On other hand, following the calculations of Brevet [39] the SHG power arising from a single surface of a $p$ NA crystal is given by, 
Here we have taken into account that a single crystal has two surfaces that will contribute coherently to the second harmonic electric field, leading to a multiplicative factor of four in right hand side of equation (3). Malagoli and Munn [19] predict that the dominant quadratic surface susceptibility tensor element for a $p$ NA surface oriented along the (202) direction is $\chi_{y y y}^{(2 s)}=1.93 \times 10^{-22} \mathrm{~m}^{2} / \mathrm{V}$, more than five orders of magnitude lower than the above estimate. This would seem to rule out simple surface SHG as the sole explanation for the observed SHG signal.

A possible alternative mechanism is that the strong electric fields applied during the electrospinning process manage to align a significant number of individual $p$ NA molecules during the ejection process and this orientation is frozen into the nanofibres during the rapid solidification that occurs as the fibres travel from the needle to the collection drum. This possibility is consistent with the Raman spectra that indicate the majority of $p$ NA molecules find themselves effectively in a liquid state dispersed within a polar environment. In many ways this process might be viewed as an accelerated form of electric field poling. This would then allow one to make use of the high molecular polarizability of individual $p$ NA molecules.

Assuming, for the sake of argument, that all the embedded $p$ NA molecules were appropriately aligned by the applied electric field, the order of magnitude of the resultant second order susceptibility can be easily estimated through the relation,

$$
\varepsilon_{0} \chi_{p N a, e f f}^{(2)}(-2 \omega ; \omega, \omega)=f(2 \omega) f^{2}(\omega) N\langle\beta(-2 \omega ; \omega, \omega)\rangle
$$


Here $N$ is the number density of $p$ NA molecules, while $\langle\beta\rangle$ is the average value of first molecular hyperpolarizability. The quantities $f(2 \omega)$ and $f(\omega)$ represent the local field factors due to the presence of the surrounding PMMA matrix. Within the Lorentz approximation these factors can be estimated according to the relation

$$
f(\omega)=\left(\varepsilon_{\omega}+2\right) / 3
$$

Together they result in an overall increase by a factor of 1.24 as estimated using the tabulated refractive indices of PMMA at the fundamental (1.484) and second harmonic (1.502) wavelengths. In this case the second harmonic generation mimics that of a crystalline material with the effective nonlinear coefficient $d_{\text {eff }}$ in equation (2) replaced by $f(2 \omega) f^{2}(\omega) N\langle\beta\rangle / \varepsilon_{0}$. Assuming that the density of $p$ NA molecules in the nanofibres is approximately that of $p \mathrm{NA}$ in the crystalline phase $\left(6.3 \times 10^{27} \mathrm{~m}^{-3}\right)$ and disregarding the possible differences in the refractive indices, we can estimate the value of the mean molecular hyperpolarizability that would be required to generate the magnitude of SHG signals we observe,

$$
\left\langle\beta_{e f f}^{p N a}\right\rangle=\frac{\varepsilon_{0}}{f(2 \omega) f^{2}(\omega) N_{p N a}}\left(\frac{L_{K D P}}{t_{p N a}}\right) \sqrt{\frac{\mathcal{P}_{2 \omega}^{p N a}}{3 \mathcal{P}_{2 \omega}^{K D P}}} d_{e f f}^{K D P}
$$

Where $t$ is the thickness of a typical $p$ NA nanofibre, roughly $800 \mathrm{~nm}$ and we have taken into account the reduction in SHG efficiency of the KDP crystal by a factor of 3 due to spatial and temporal beam walkoff. Substituting the appropriate values into the above equation, we find that $\left\langle\beta_{\text {eff }}^{p N a}\right\rangle \approx 7 \times 10^{-50} \mathrm{Cm}^{3} / \mathrm{V}^{2}$. Huyskens et al. [40] have measured the average first hyperpolarizability of $p$ NA in a variety of solvents. From their extensive list, the solvent that is/chemically most similar to PMMA is methyl acetate, for which they measure a value of $9.5 \times 10^{-50} \mathrm{Cm}^{3} / \mathrm{V}^{2}$ at an incident wavelength of $1064 \mathrm{~nm}$ when corrected for the most recent value based on second harmonic generation in quartz [41]. This agrees with the above order of magnitude estimate, suggesting that a large fraction of the second order optical response of the individual $p$ NA molecules was translated into a macroscopic response of the doped electro-spun polymeric fibres.

The equivalent effective second order susceptibility can be estimated to be $\chi_{\text {eff }}^{(2)} \approx 65 \mathrm{pm} / \mathrm{V}$, close to that estimated for $p$ NA PLLA elctrospun fibres [10] and the same magnitude as was reported by Miyazaki et al.[42] for guest-host mixtures of $p \mathrm{NA}$ and $\operatorname{poly}(\varepsilon$ - caprolactone). However, our electro-spinning method has the added advantage of producing fibres with a well-defined alignment and good optical quality leading to a coherent and strongly polarized response. 


\section{Conclusions}

In this study, we have shown it is possible to obtain a strong macroscopic second harmonic response from $p$ NA molecules embedded in PMMA electro-spun fibres with sub-micron diameters. Low deposition rates favour stronger SHG responses. The magnitude of the effect indicates that it is not simply a result of symmetry breaking at the $p$ NA-polymer surfaces, but rather suggests that a non-centrosymmetric arrangement has been induced, possibly as the result of strong hydrogen bonds between the $p$ NA molecules and the polymeric host. We speculate that the high electric fields experienced during extrusion in the electro-spinning process, coupled to the unusually large ground state $\mathrm{p} N A$ electric dipole moment of nearly 7 Debye may lead to highly aligned molecular structures that are subsequently frozen into the 1D nanostructure by the rapid solidification that occurs. This would in essence, amount to a highly accelerated version of electric field poling commonly done to align organic molecules with strong nonlinear responses in polymer substrates. However contrary to normal electric field poling, the electrospun fibres retain their ability to generate significant second harmonic light over timescales extending to one year.

The electro-spinning technique should be extendable to other organic molecules with large dipole moments and strong second order nonlinear optical responses. Given the large number of NLO active organic compounds that crystallize in centrosymmetric structures; this technique has the potential to open up a new pathway to fabricate sub-wavelength photonic materials with large second order nonlinear optical responses.

\section{Acknowledgements}

Hugo Gonçalves thanks the Portuguese Foundation for Science and Technology (FCT) for the support under grant $\mathrm{PD} / \mathrm{BD} / 111873 / 2015$. The equipment used to characterize the second harmonic response of the electro-spun fibres was acquired within the framework of the Portuguese National Program for Scientific Re-equipment, contract REEQ-25/FIS/2005 with funds from POCI 2010 (FEDER) and FCT. The Raman microscope was acquired through the project nSTeP Nanostructured Systems for Tailored Performance, NORTE-07-0124-FEDER-000039, ON.2. This work was in part developed in the scope of the projects CICECO - Aveiro Institute of Materials (UID/CTM/50011/2013), financed by national funds through the Fundação para a Ciência e a Tecnologia/Ministério da Educação e Ciência (FCT/MEC) and co-financed by FEDER under the PT2020 Partnership Agreement. The authors are grateful to A. M. P. Botas (University of Aveiro) for help in acquiring the hyperspectral microscopy data. 


\section{References}

[1] Garmire E 2013 Nonlinear optics in daily life Opt. Express $2130532-44$

[2] Rosenne S, Grinvald E, Shirman E, Neeman L, Dutta S, Bar-Elli O, Ben-Zvi R, Oksenberg E, Milko P, Kalchenko V, Weissman H, Oron D and Rybtchinski B, 2015 Self-assembled organic nanocrystals with strong nonlinear optical response Nano Lett. 15 7232-37

[3] Xu J, Semin S, Niedzialek D, Kouwer PHJ, Fron E, Coutino E, Savoini M, Li Y, Hofkens J, Uji-I H, Beljonne D, Rasing T and Rowan AE 2013 Self-assembled organic microfibers for nonlinear optics Adv. Mater. $252084-9$

[4] Ray PC and Leszczynski $2006 \mathrm{~J}$ Nonlinear optical properties of highly conjugated push-pull porphyrin Aggregates: role of intermolecular interaction Chem. Phys. Lett. 419 578-83

[5] Jeon S, Malyarchuk V, Rogers JA and Wiederrecht, GP Fabricating three-dimensional nanostructures using two photon lithography in a single exposure step Opt. Express 14 2300-08

[6] Koh AL, Fernández-Domínguez AI, McComb DW, Maier SA and Yang JKW 2011 Highresolution mapping of electron-beam-excited plasmon modes in lithographically defined gold nanostructures Nano Lett. 11 1323-30

[7] Shambat G, Ellis B, Petykiewicz J, Mayer M; Majumdar A, Sarmiento T, Harris J, Haller EE, and Vuckovic J 2012 Electrically driven photonic crystal nanocavity devices IEEE J. Sel. Top. Quantum Electron. 18 1700-10

[8] Isakov DV., de Matos Gomes E, Vieira LG, Dekola T, Belsley MS and Almeida BG 2011 Oriented single-crystal-like molecular arrangement of optically nonlinear 2-methyl-4-nitroaniline in electrospun nanofibers ACS Nano 5 73-8

[9] Bhardwaj N and Kundu SC 2010 Electrospinning: a fascinating fiber fabrication technique Biotechnol. Adv. 28 325-47

[10] Isakov DV, Belsley MS, de Matos Gomes E, Gonçalves H, Schellenberg P and Almeida B G 2014 Intense optical second harmonic generation from centrosymmetric nanocrystalline para-nitroaniline Appl. Phys. Lett. 104181903

[11] Isakov D, de Matos Gomes E, Belsley MS, Almeida B and Cerca N 2012 Strong enhancement of second harmonic generation in 2-methyl-4-nitroaniline nanofibers Nanoscale 44978

[12] Chronakis IS 2005 Novel nanocomposites and nanoceramics based on polymer nanofibers using electrospinning process - a review J. Mater. Process. Technol. 167 283-93

(13) Li J, Li H, Hu H, Zhao Y and Wang Q 2015 Preparation and application of polymer nano-fiber doped with nano-particles Opt. Mater. $4049-56$

[14] Ren ML, Liu W, Aspetti CO, Sun L and Agarwal R 2014Enhanced second-harmonic Generation from metal-integrated semiconductor nanowires via highly confined whispering gallery modes Nat. Commun. 55432

[15] Kravets VG, Marshall OP, Nair, RR, Thackray B, Zhukov A, Leng J and Grigorenko AN 2015 Engineering optical properties of a graphene oxide metamaterial assembled in microfluidic channels Opt. Express 231265 
[16] Verbiest T, Houbrechts S, Kauranen M, Clays K and Persoons A 1997 Second-order nonlinear optical materials: recent advances in chromophore design J. Mater. Chem. 7 2175-89

[17] Shkir M, Riscob B, Hasmuddin M, Singh P, Ganesh V, Wahab MA, Dieguez E and Bhagavannarayana G 2014 Optical spectroscopy, crystalline perfection, etching and mechanical studies on p-nitroaniline (pNA) single crystals Opt. Mater. (Amst). 36 675-81

[18] Kobayashi H and Kotani M 1994 Study of single crystal surface with second-harmonic generation: p -nitroaniline Mol. Cryst. Liq. Cryst. Sci. Technol. Sect. A. Mol. Cryst. Liq. Cryst. 252 277-81

[19] Malagoli M and Munn, RW 2000 Microscopic calculation of surface-induced second-harmonic generation in crystals of para-nitroaniline J. Chem. Phys. 112 6757-62

[20] Marchewka MK, Ratajczak H and Debrus S 2003 Infrared spectrum and nonlinear optical properties of p-nitroaniline-1-tartaric acid (2:1) molecular complex J. Nonlinear Opt. Phys. Mater. $12113-21$

(21) Kosenkov D and Slipchenko LV. 2011 Solvent effects on the electronic transitions of pnitroaniline: a QM/EFP study J. Phys. Chem. A 115 392-401

[22] Kuzyk M G, Pérez-Moreno J and Shafei S 2013 Sum rules and scaling in nonlinear optics Physics Reports. 529 297-398

[23] Kato M, Kiguchi M, Sugita N and Taniguchi Y 1997 Second-order nonlinearity of mixtures Including p-nitroaniline derivatives J. Phys. Chem. B, $1018856-59$

[24] Cambré S, Campo J, Beirnaert C, Verlackt C, Cool P and Wenseleers W 2015 Asymmetric dyes align inside carbon nanotubes to yield a large nonlinear optical response Nat. Nanotechnol. $10248-$ 52

[25] Zhang CL and Yu SH 2014 Nanoparticles meet electrospinning: recent advances and future prospects Chem. Soc. Rev. $\mathbf{4 3} 4423-48$

[26] Garreau A and Duvail JL 2014 Recent advances in optically active polymer-based nanowires and nanotubes Adv. Opt. Mater. $21122-40$

[27] Bernardo C, Belsley M, de Matos Gomes E, Gonçalves H, Isakov D, Liebold F, Pereira E, Pires V, Samantilleke A, Vasilevskiy M and Schellenberg P 2014 A versatile fluorescence lifetime imaging system for scanning large areas with high time and spatial resolution Second International Conference on Applications of Photonics and Optics, Proc. SPIE vol 9286 (Aveiro, Portugal, 2630 May 2014) eds. Martins Costa MFPC and Nogueira, RN (Bellingham SPIE) pp 928637-8

[28] Piperno S, Lozzi L, Rastelli R, Passacantando M and Santucci S 2006 PMMA nanofibers production by electrospinning Appl. Surf. Sci. 252 5583-6

[29] Zak AK, Majid, WHA, Abrishami ME and Yousefi R 2011 X-Ray analysis of ZnO nanoparticles by Williamson-Hall and size-strain plot methods Solid State Sci. 13 251-6

[30] Varsányi G 1969 Vibrational Spectra of Benzene Derivatives, 1st edition (New York, Academic Press) 1969

[31] Marlow F, Hill W, Caro J and Finger G 1993 Raman-study on p-nitroaniline in channels of the molecular-sieve alpo(4)-5 J. Raman Spectrosc. 24 603-8 
[32] Harrand M 1975 Raman study on para nitroaniline single crystal. II: internal vibrations J. Raman Spectrosc. 4 53-73

[33] Harrand M 1979 Raman study on p-nitroaniline: molecular structure in the molten phase J. Raman Spectrosc. 8 161-4

[34] Brasselet S, le Floc'h V, Treussart F, Roch, JF, Zyss J, Botzung-Appert E and Ibanez A 2004 In situ diagnostics of the crystalline nature of single organic nanocrystals by nonlinear microscopy. Phys. Rev. Lett. 92 207401-1

[35] Boyd, RW 2003 Nonlinear Optics 3rd. ed. (Rochester Academic Press)

[36] Nikogosyan DN 1991 Handbook of Nonlinear Optical Crystals, 3rd Revised ed. (Berlin, Springer Verlag)

[37] SNLO nonlinear optics code available from A. V. Smith, AS-Photonics, Albuquerque, NM

[38] Wang H and Weiner AM 2003 Efficiency of short-pulse type I second harmonic generation with simultaneous spatial walk-off, temporal walk-off and pump depletion IEEE J. Quantum Electronics 391600

[39] Brevet PF 1996 Phenomenological three-layer model for surface second-harmonic generation at the interface between two centrosymmetric media J. Chem. Soc. Faraday Trans. 924547

[40] Huyskens FL, Huyskens PL and Persoons AP 1998 Solvent dependence of the first hyperpolarizability of p-nitroanilines: differences between nonspecific dipole-dipole interactions and solute-solvent H-bonds J. Chem. Phys. 1088161

[41] Reis H 2006 Problems in the comparison of theoretical and experimental hyperpolarizabilities revisited J. Chem. Phys. 125014506

[42] Miyazaki T, Wanntabe T, and Miyata S 1988 Highly efficient second harmonic generation in pnitroanaline / poly(lactone) systems Japanese J. of Appl. Phys. 27 L1724 